\title{
Volume Delineation in Cervical Cancer With T2 and Diffusion- weighted MRI: Agreement on Volumes Between Observers
}

\author{
CONSUELO ROSA ${ }^{1,2}$, ANDREA DELLI PIZZI ${ }^{2,3}$, ANTONIETTA AUGURIO ${ }^{1}$, LUCIANA CARAVATTA ${ }^{1}$, \\ MONICA DI TOMMASO ${ }^{1}$, ERICA MINCUZZI ${ }^{3}$, SEBASTIANO CINALLI $^{4}$, \\ RAFFAELLA BASILICO ${ }^{3}$, ANNAMARIA PORRECA ${ }^{5}$, MARTA DI NICOLA $^{6}$ and DOMENICO GENOVESI ${ }^{1,2}$ \\ ${ }^{1}$ Department of Radiation Oncology, SS. Annunziata Hospital, G. D’Annunzio University, Chieti, Italy; \\ ${ }^{2}$ Department of Neuroscience, Imaging and Clinical Sciences, G. D'Annunzio University, Chieti, Italy; \\ ${ }^{3}$ Department of Radiology, SS. Annunziata Hospital, G. D’Annunzio University, Chieti, Italy; \\ ${ }^{4}$ Division of Pathology, SS. Annunziata Hospital, Chieti, Italy; \\ ${ }^{5}$ Department of Economy, G. D’Annunzio University, Chieti, Italy; \\ ${ }^{6}$ Laboratory of Biostatistics, Department of Medical, Oral and Biotechnological Sciences, \\ G. D’Annunzio University, Chieti, Italy
}

\begin{abstract}
Aim: To delineate cervical cancer gross tumor volume (GTV) on T2-magnetic resonance imaging (MRI) and apparent diffusion coefficient (ADC) maps, assessing volumes and inter-observer agreement between two observers. Patients and Methods: A radiologist and a radiation oncologist delineated GTV on $T 2$ (T2 $\left.2_{G T V}\right)$ and $A D C$ (ADC $\left.C_{G T V}\right)$ sequences. Dice similarity index (DICE) and Bland-Altman analysis were used to estimated concordance. Results: Mean $T 2_{G T V}$ and $A D C_{G T V}$ volumes were $43.84 \pm 71.47 \mathrm{cc}$ and $37.28 \pm 68.92 \mathrm{cc}$ according to the radiologist, and $43.4 \pm 70.44$ cc and $36.65 \pm 69.21 \mathrm{cc}$ according to the radiation oncologist. $A D C$ led to statistically significantly smaller volumes compared to T2. The mean DICE index was 0.86 for T2 $G T V$ and 0.84 for $A D C_{G T V}$. The Bland-Altman plots globally showed concordance. Conclusion: GTV delineation was smaller in the ADC maps compared to T2-MRI, reaching an almost perfect agreement between observers. Thanks to this acceptable variability, adding functional imaging might provide more information for tumor delineation, improving reproducibility for image-guided adaptive radiotherapy.
\end{abstract}

Nowadays, the mainstay of treatment for locally advanced cervical cancer (LACC) includes external beam radiotherapy

This article is freely accessible online.

Correspondence to: Consuelo Rosa, Department of Radiation Oncology, SS Annunziata Hospital, Via Dei Vestini, 66100 Chieti, Italy. Tel: +39 0871358244, Fax: +39 08713557374, e-mail: c.rosa155@gmail.com.

Key Words: Cervical cancer, inter-observer agreement, magnetic resonance imaging, radiotherapy.
(EBRT), concomitant chemotherapy and brachytherapy. T2weighted magnetic resonance imaging (MRI) is the imaging modality to be preferred for careful and accurate primary staging of the tumor $(1,2)$.

Despite the extensive use of MRI, recent studies reported significant uncertainties in T2-weighted MRI delineation, describing a poor interobserver variability in brachytherapy target volume, represented by conformity indices of $0.58-0.60$ for gross tumor volume (GTV) at brachytherapy, and 0.39-0.79 (mostly between 0.70-0.79) for high-risk clinical target volume $(3,4)$. Considering this aspect and the possible advantages of improved diagnostic techniques in a dose-intensification strategy, in particular in patients with higher risk of local relapse, its integration with diffusion-weighted MRI (DWI) might improve tumor volume definition in addition to patient outcome. In this way, a recent study demonstrated the accuracy of the apparent diffusion coefficient (ADC) map obtained at 2 weeks during radiotherapy in allowing an early prediction of treatment response (5).

DWI-MRI is considered an encouraging functional imaging mode in the definition of a biological target volume, thanks to its capability for detecting highly metabolic regions. DWI-MRI evaluates the random motion of water molecules, mostly present in the extracellular space: compared to normal tissue, in cervical cancer, as in other neoplasms, tumor is densely cellular, showing restricted diffusion. This distribution is quantitatively well measured by ADC. In DWI, cancer has an increased signal intensity, which results in a reduction of the corresponding ADC map. It is well established that both DWI techniques as well as ADC measurement improves cervical cancer clinical assessment, regarding both tumor and nodal status, and histology (6). Few studies have analyzed the use of DWI for image-guided radiotherapy in cervical cancer (7). 
Since T2 MRI has been suggested by the Groupe Européen de Curiethérapie - European Society for Radiotherapy and Oncology (GEC-ESTRO) guidelines for target delineation in image-guided adaptive brachytherapy, and DWI is able to define a biological target volume, we conducted a retrospective study in order to assess differences in the definition of the GTV based on T2-weighted and ADC maps. In detail, we estimated volumes and interobserver agreement between two observers, a radiologist and a radiation oncologist, in order to improve volume delineation in patients with cervical cancer.

\section{Patients and Methods}

We retrospectively evaluated 37 patients, with a median age of 55 years (range $=28-94$ years), treated at our Radiotherapy Department from January 2013 to July 2018. All patients had adenocarcinoma or squamous cell carcinoma of the cervix and they underwent diagnostic 1.5T MRI prior to radiotherapy. According to the International Federation of Gynecology and Obstetrics classification, two (5.4\%) patients were staged with IB1 disease, six (16.2\%) with IIA 1,17 (46\%) with IIB, two (5.4\%) with IIIB and 10 (27\%) with IVA (8). All patients were treated with external beam radiotherapy, with a total dose of 45-50 Gy (1.8 Gy/day). Concomitant chemotherapy consisted of weekly cisplatin at $40 \mathrm{mg} / \mathrm{m}^{2}$.

A radiologist and a radiation oncologist independently delineated the whole GTV on T2 (T2 $\left.{ }_{\mathrm{GTV}}\right)$ and $\mathrm{ADC}$ map $\left(\mathrm{ADC}_{\mathrm{GTV}}\right)$ axial sequences on RayStation platform (RaySearch Laboratories, Stockholm, Sweden). They had the possibility of adjusting window and level settings. The tumor appeared as a hypo-intense signal on the ADC map, created by the corresponding DWI. The MRI was not co-registered with the computed tomographic (CT) scan simulation.

Statistical analysis. $\mathrm{T}_{\mathrm{GTV}}$ and $\mathrm{ADC}_{\mathrm{GTV}}$ delineated by the two observers were subjected to statistical analysis, and reported as the mean and standard deviation. The deviation from a normal distribution for each parameter was evaluated by Shapiro-Wilk's test. $2_{\mathrm{GTV}}$ and $\mathrm{ADC}_{\mathrm{GTV}}$ were compared using the $t$-test. A $p$-value of less than 0.05 was considered statistically significant. Dice similarity index (DICE) (9), Bland-Altman (10) analysis (mean difference, $95 \%$ limits of agreement), coefficient of repeatability (CR) and intra-class correlation coefficient (ICC) were used to assess agreement between the radiologist and radiation oncologist. ICC is the ratio of the intersubject component of discrepancy to the total discrepancy; better repeatability is obtained with a high ratio. The CR is the value below which the absolute differences between two measurements would lie with 0.95 probability, directly related to the $95 \%$ limits of agreement suggested by Bland and Altman. In order to identify any possible relationship between the measurement error and the true value, a concordance plot of volumes obtained by the observers against their mean was performed. The Bland-Altman method was applied to evaluate the $95 \%$ limits of agreement. The DICE index evaluated the correspondence between volumes according to the radiation oncologist compared to those according to the radiologist, considered the benchmark. Defining volumes $\mathrm{A}$ and $B$, respectively, DICE was: $\mathrm{DICE}=2 \times \mathrm{A} \cup \mathrm{B} / \mathrm{A}+\mathrm{B}$ and ranged from 0 to 1 (no overlap between volumes to complete overlap). Agreement was slight when DICE was between 0 to 0.2 , fair between 0.2 to 0.4 , moderate 0.4 to 0.6 , substantial 0.6 to 0.8 and
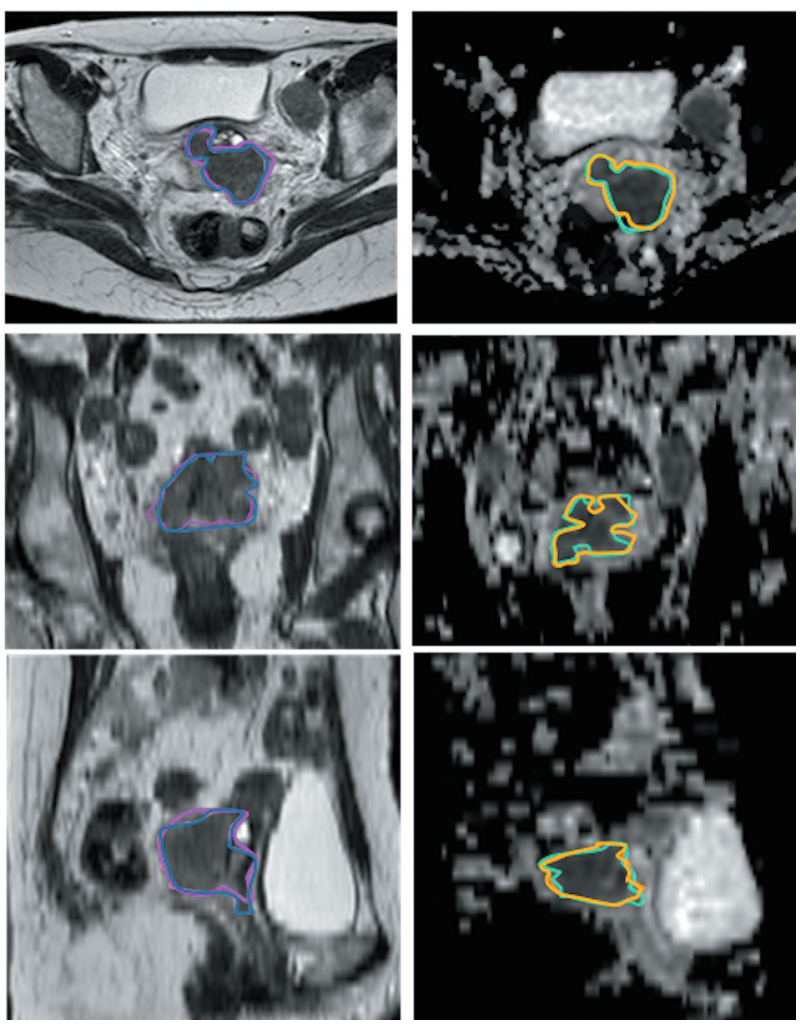

Figure 1. Gross tumor volume (GTV) representations on T2-weighted (left) and apparent diffusion coefficient (ADC) maps (right) on axial (upper), coronal (middle) and sagittal (lower) magnetic resonance imaging planes. Pink contour: radiologist $T 2_{G T V}$; blue contour: radiation oncologist $T 2_{G T V}$; orange contour: radiologist $A D C_{G T V}$; light green contour: radiation oncologist $A D C_{G T V}$.

perfect with DICE from 0.8 to 1 . Statistical analysis was performed using the $\mathrm{R}$ environment.

\section{Results}

Comparison between volumes. Each observer analyzed 37 MRIs. Mean $\mathrm{T}_{\mathrm{GTV}}$ and $\mathrm{ADC}_{\mathrm{GTV}}$ volumes were $43.84 \pm 71.47 \mathrm{~cm}^{3}$ and $37.28 \pm 68.92 \mathrm{~cm}^{3}$ for the radiologist, and $43.4 \pm 70.44 \mathrm{~cm}^{3}$ and $36.65 \pm 69.21 \mathrm{~cm}^{3}$ for the radiation oncologist. With a statistically significant difference $(p<0.001$ for both the radiologist and radiation oncologist), values for $\mathrm{T} 2_{\mathrm{GTV}}$ were larger compared to those for $\mathrm{ADC}_{\mathrm{GTV}}$.

Comparison between radiologist and radiation oncologist measurements. Figure 1 show an example of $\mathrm{T} 22_{\mathrm{GTV}}$ and $\mathrm{ADC}_{\mathrm{GTV}}$ MRI. Concordance plots for $\mathrm{T}_{\mathrm{GTV}}$ and $\mathrm{ADC} \mathrm{GTV}_{\mathrm{GTV}}$ between observers are shown in Figure 2. The mean DICE index values for $\mathrm{T}_{\mathrm{GTV}}$ and $\mathrm{ADC}_{\mathrm{GTV}}$ were $0.86 \pm 0.06$ and $0.84 \pm 0.08$, respectively. The ICC for T2 ${ }_{\mathrm{GTV}}$ was 0.998 [95\% confidence intervaI $(\mathrm{CI})=0.997-0.999]$ and $0.999(95 \%$ 

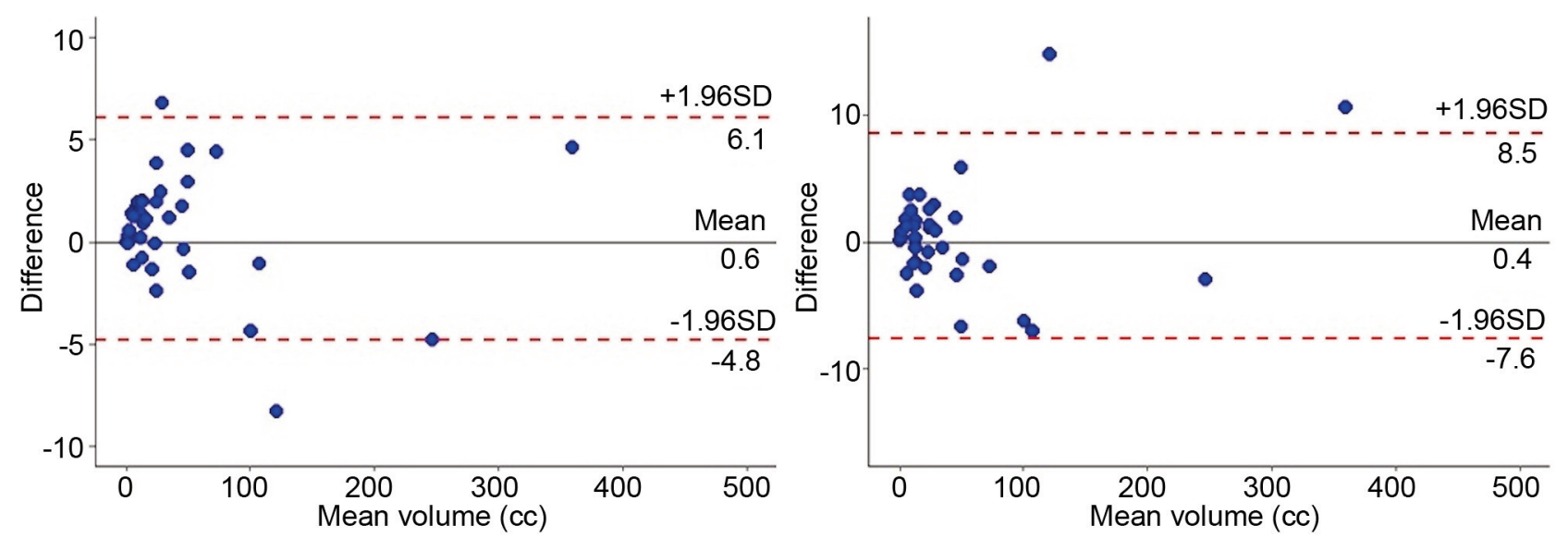

Figure 2. Bland-Altman concordance plot for apparent diffusion coefficient gross tumor volume $\left(A D C_{G T V}\right)$ and T2-weighted gross tumor volume $\left(T 2_{G T V}\right)$ by the radiologist and the radiation oncologist. The upper and lower $95 \%$ limits of agreements are represented as dotted lines; the mean difference is represented as a continuous line. SD: Standard deviation.

$\mathrm{CI}=0.998-1.000)$ for $\mathrm{ADC}_{\mathrm{GTV}}$, respectively. The $\mathrm{CR}$ was $5.496(95 \% \mathrm{CI}=4.481-7.110)$ for $\mathrm{T}_{\mathrm{GTV}}(p=0.516)$ and 7.984 $(95 \% \mathrm{CI}=6.509-10.329) \quad(p=0.173)$ for $\mathrm{ADC}_{\mathrm{GTV}}$, respectively. The Bland-Altman plots show some outliers beyond the limits of agreements but globally the methods for the two observers were in concordance, conforming to the CR coefficients reported above.

\section{Discussion}

We conducted this retrospective study with the aim of comparing the GTV delineated on T2-MRI with the GTV defined on the ADC maps, in order to estimate the difference in volume and volume reproducibility between different observers. It is known that ADC maps, in addition to T2weighted sequences, can improve the accuracy of disease detection and predict treatment response. A new interesting and useful function might also be its use in tumor delineation.

Nowadays, external beam radiotherapy and brachytherapy represent the major modalities for cervical cancer treatment. In this setting, MRI is crucial in treatment planning. In fact, the GTV defined for intracavitary brachytherapy is obtained by clinical examination and T2-MRI.

3D CT and MRI are standardly used both for GTV coverage and evaluation of the dose to organs at risk (OARs), in particular for brachytherapy. Many authors had found correlations between late radiation toxicities and total dose given both to the rectum and bladder, as well as other studies had tried to give a possible comparison between late radiation toxicities and volumetric doses (11-14).

The International Federation of Gynecology and Obstetrics classification underlines the role of imaging in cervical cancer staging, and several studies have shown that the only clinical examination is not sufficient, indeed taking advantage by adding MRI (15-17).

MRI is the gold standard for tumor staging due to its excellent soft-tissue contrast. In fact, MRI techniques are useful for assessing parametrial invasion, detecting lymph node metastases and determining tumor sub-type and grading (18), in addition to the appropriate visualization of OARs such as the bladder, vagina, rectum and sigmoidal colon (2). Furthermore, with the development of DWI and dynamic contrast-enhanced MRI (DCE-MRI), the tumor microenvironment can be investigated better than using conventional imaging alone.

The Gynecological GEC-ESTRO Working Group gave recommendations for MRI-based adaptive target volume as well as for OARs (2). In addition, ADC values were considered a quantitative tool not only for diagnosis but also for the evaluation of treatment response in cervical cancer (19), as in other malignancies (20-22).

It is well known that DWI can improve treatment response assessment in cervical cancer (19). In fact, it has the potential to discriminate between normal tissue and a neoplasm. Moreover, it may provide information about the histological type of cervical cancer (23). The sensitivity and specificity of DWI in cervical cancer detection were about 94-100\% and $85 \%$, respectively, as demonstrated by Chen et al. (24). Concerning ADC maps, they are useful not only for prediction of treatment response but also for monitoring of early response, even if further studies are necessary.

The potential use of functional imaging, represented by DWIMRI, positron-emission tomography (PET-CT) and DCE-MRI, could improve individual dose planning. In this scenario, it could be of interest to include all the information resulting from these different modalities (25). T2-MRI, DWI-MRI and PET-CT 
imaging have been studied for target delineation in patients with LACC (26). In this scenario, we investigated the potential of DWI-MRI and the corresponding ADC maps in order to study the possibility of having more information on tumor volume.

The concordance between FDG-PET and DWI, as functional imaging, was retrospectively investigated in 20 patients with cervical cancer, comparing fluorodeoxyglucose (FDG) and ADC tumor volumes (27). The average ADC MRI volume was larger compared to FDG PET ones $\left(43.8 \mathrm{~cm}^{3} v s\right.$. $39.0 \mathrm{~cm}^{3}$ ), with a good agreement between the two modalities, assessed with DICE index (0.76 \pm 0.06$)$. Moreover, the authors demonstrated a concordance in LACC sub-volumes between increased FDG uptake and ADC restriction, with comparable FDG-PET and DWI delineation (27).

The information obtained by DWI/DCE-MRI/FDG-PET in addition to T2-MRI and interobserver variability were also confirmed by Han et al. In their study, the GTV and the high-risk clinical target volume of 22 patients with LACC were firstly contoured on T2 images and then changed using DWI/DCE-MRI/FDG-PET images, by two readers. Half of T2-GTVs were modified based on DWI/DCE-MRI/FDGPET by one observer because of both better differentiation and visualization of residual neoplasm, whereas the second observer changed GTV in 17 patients. The conformity index for GTV was significantly improved, thanks to the incorporation of functional imaging, from 0.54 (obtained on T2 imaging) to $0.65(p=0.003)$. Both readers adapted the high-risk clinical target volume in three and eight cases, reporting a better concordance with the addition of functional imaging (conformity index from 0.71 to $0.76, p=0.06$ ) (26). The authors concluded that supplementing T2-MRI with DCE-MRI/DWI/FDG-PET imaging leads to a significant reduction in interobserver variability.

Starting from the improvement of functional imaging in the accuracy of disease detection and prediction of treatment response, and the new evidence in its useful tumor delineation, we analyzed its role in ADC maps in comparison to T2-MRI.

Similarly with previous studies performed on other tumor types (28-30), our purpose was that of comparing the GTV delineated on T2-MRI with the GTV defined on the ADC maps. A previous retrospective study conducted on 15 patients with cervical cancer obtained T2 volumes generally larger than those on ADC $\left(64.0 \pm 70.5 \mathrm{~cm}^{3}\right.$ vs. $52.3 \pm 54.6 \mathrm{~cm}^{3}$, $p=0.0074$, sign test) (7). Similarly, our study on 37 patients reported $\mathrm{ADC}_{\mathrm{GTV}}$ smaller compared to $\mathrm{T} 2_{\mathrm{GTV}}$, for both observers, a radiologist and a radiation oncologist, reporting mean $\mathrm{T} 2{ }_{\mathrm{GTV}}$ and $\mathrm{ADC}_{\mathrm{GTV}}$ volumes of $43.84 \pm 71.47 \mathrm{~cm}^{3}$ and $37.28 \pm 68.92 \mathrm{~cm}^{3}$ for the radiologist, and $43.4 \pm 70.44 \mathrm{~cm}^{3}$ and $36.65 \pm 69.21 \mathrm{~cm}^{3}$ for the radiation oncologist, respectively. Furthermore, as some authors compared volumes on T2-MRI and PET-CT, and reported areas on PET-CT undetected on T2MRI, ADC maps might highlight areas hidden on T2 images, in particular regarding smaller target volumes (31).
Differently from previous authors, who did not investigate a concordance in volume definition between different observers, we reported an agreement between volumes obtained by a radiologist and a radiation oncologist, with a mean DICE index for T2 $\mathrm{GTV}$ of $0.86 \pm 0.06$ and $0.84 \pm 0.08$ for $\mathrm{ADC}_{\mathrm{GTV}}$, respectively, indicating an almost perfect agreement. This almost perfect agreement between different observers could suggest as not only T2-weighted images but also ADC map might well be used for volume definition by radiation oncologists.

Our study had some limitations. First of all, it was a retrospective study with a small study cohort. For this reason, further studies, possibly prospective and multicentric, are warranted to confirm our results. Secondly, we used only T2-weighted images and ADC maps for functional target volume delineation. It might be of interest to compare GTV delineation on T2 images to both ADC maps and FDG$\mathrm{PET} / \mathrm{CT}$ images, in order to evaluate whether biological imaging might better identify smaller target volumes. Future evaluations should also be made on both ADC maps and T2MRI in order to evaluate the accuracy of GTV delineation, as well as including PET-CT for delineation.

In conclusion, in our study, significantly smaller volumes were obtained for the GTV when using ADC maps compared to T2-weighted MRI. This might suggest that adding functional imaging such as ADC maps obtained from DWI might give more information for tumor delineation. Furthermore, the DICE index reported an almost perfect agreement for $\mathrm{T} 2_{\mathrm{GTV}}$ and $\mathrm{ADC}_{\mathrm{GTV}}$ obtained by a radiologist and a radiation oncologist, demonstrating that target contouring on ADC maps might represent a successful option with an acceptable variability between different observers. Further studies with a larger study population are warranted to confirm these promising results. This could be investigated in order to improve delineation reproducibility for image-guided adaptive radiotherapy, even with a view to reducing doses to adjacent OARs. In addition, FDG-PET imaging might be taken into consideration in order to provide further functional tumor information.

\section{Conflicts of Interest}

The Authors report no conflicts of interest.

\section{Authors' Contributions}

CR, ADP, AA, LC, MDT and DG designed and coordinate the study and the analysis. CR, ADP, EM and SC collected the data. AA, LC, MDT and RB reviewed and approved data selection. CR, ADP, AA, LC and MDT performed main data analysis and provided pictures elaboration. AP and MDN performed statistical data analysis. CR, ADP, AA and MDT drafted the article. LC and DG critically revised the study and the article. All Authors reviewed and approved the final article. 


\section{References}

1 Haie-Meder C, Potter R, Van Limbergen E, Briot E, De Brabandere M, Dimopoulos J, Dumas I, Hellebust TP, Kirisits C, Lang S, Muschitz S, Nevinson J, Nulens A, Petrow P, WachterGerstner N, Gynaecological (GYN) GEC-ESTRO Working Group: Recommendations from Gynaecological (GYN) GEC-ESTRO Working Group (I): Concepts and terms in 3D image based 3D treatment planning in cervix cancer brachytherapy with emphasis on MRI assessment of GTV and CTV. Radiother Oncol 74: 235245, 2005. PMID: 15763303. DOI: 10.1016/j.radonc.2004.12.015

2 Dimopoulos JC, Petrow P, Tanderup K, Petric P, Berger D, Kirisits C, Pedersen EM, van Limbergen E, Haie-Meder C and Pötter R: Recommendations from Gynaecological (GYN) GECESTRO Working Group (IV): Basic principles and parameters for MR imaging within the frame of image based adaptive cervix cancer brachytherapy. Radiother Oncol 103: 113-122, 2012. PMID: 22296748. DOI: 10.1016/j.radonc.2011.12.024

3 Petric P, Hudej R, Rogelj P, Blas M, Tanderup K, Fidarova E, Kirisits C, Berger D, Dimopoulos JCA, Pötter R and Hellebust TP: Uncertainties of target volume delineation in MRI guided adaptive brachytherapy of cervix cancer: A multi-institutional study. Radiother Oncol 107: 6-12, 2013. PMID: 23453539. DOI: 10.1016/j.radonc.2013.01.014

4 Dimopoulos JC, De Vos V, Berger D, Petric P, Dumas I, Kirisits C, Shenfield CB, Haie-Meder C and Richard Pötter R: Interobserver comparison of target delineation for MRI-assisted cervical cancer brachytherapy: Application of the GYN GECESTRO recommendations. Radiother Oncol 91: 166-172, 2009. PMID: 19062118. DOI: 10.1016/j.radonc.2008.10.023

5 Liu Y, Sun H, Bai R and Ye Z: Time-window of early detection of response to concurrent chemoradiation in cervical cancer by using diffusion-weighted MR imaging: A pilot study. Radiat Oncol 10: 185, 2015. PMID: 26337954. DOI: 10.1186/s13014-015-0493-6

6 Punwani S: Diffusion weighted imaging of female pelvic cancers: concepts and clinical applications. Eur J Radiol 78: 2129, 2011. PMID: 20801592. DOI: 10.1016/j.ejrad.2010.07.028

7 Esthappan J, Ma DJ, Narra VR, Raptis CA and Grigsby PW: Comparison of apparent diffusion coefficient maps to T2weighted images for target delineation in cervix cancer brachytherapy. J Contemp Brachytherapy 3: 193-198, 2011. PMID: 23346128. DOI: $10.5114 /$ jcb.2011.26470

8 Pecorelli S, Zigliani L and Odicino F: Revised FIGO staging for carcinoma of the cervix. Int J Gynaecol Obstet 105: 107-108, 2009. PMID: 19342051. DOI: 10.1016/j.ijgo.2009.02.009

9 Fotina I, Lütgendorf-Caucig C, Stock M, Pötter R and Georg D: Critical discussion of evaluation parameters for inter observer variability in target definition for radiation therapy. Strahlenther Onkol 188: 160-167, 2012. PMID: 22281878. DOI: 10.1007/s00066-011-0027-6

10 Kouwenhoven E, Giezen M and Struikmans H: Measuring the similarity of target volume delineations independent of the number of observers. Phys Med Biol 54: 2863-2873, 2009. PMID: 19384002. DOI: 10.1088/0031-9155/54/9/018

11 Hashim N, Jamalludin Z, Ung NM, Ho GF, Malik RA and Phua VCE: CT based 3-dimensional treatment planning of intracavitary brachytherapy for cancer of the cervix: comparison between dose-volume histograms and ICRU point doses to the rectum and bladder. Asian Pac J Cancer Prev 15: 5259-5264, 2014. PMID: 25040985. DOI: 10.7314/apjcp.2014.15.13.5259
12 Yang L and Lv Y: Possible risk factors associated with radiation proctitis or radiation cystitis in patients with cervical carcinoma after radiotherapy. Asian Pac J Cancer Prev 13: 6251-6255, 2012. PMID: 23464440. DOI: 10.7314/apjcp.2012.13.12.6251

13 Tan LT, Coles CE, Hart C and Tait E: Clinical impact of computed tomography-based image-guided brachytherapy for cervix cancer using the tandem-ring applicator - The Addenbrooke's Experience. Clin Oncol 21: 175-172, 2009. PMID: 19101130. DOI: 10.1016/j.clon.2008.12.001

14 Srivastava S, Painuly NK, Mishra SP, Srivastava K, Singh N and Bhatt MLB: Evaluation of volumetric doses of organs at risk in carcinoma cervix patients with HDR intracavitary brachytherapy and comparison of CT-based and conventional plan. J Biomed Phys Eng 9: 603-612, 2019. PMID: 32039090. DOI: 10.31661/ jbpe.v0i0.867

15 Mitchell DG, Snyder B, Coakley F, Reinhold C, Thomas G, Amendola M, Schwartz LH, Woodward P, Pannu H and Hricak H: Early invasive cervical cancer: Tumor delineation by magnetic resonance imaging, computed tomography, and clinical examination, verified by pathologic results, in the ACRIN 6651/GOG 183 Intergroup Study. J ClinOncol 24: 5687-5694, 2006. PMID: 17179104. DOI: 10.1200/JCO.2006.07.4799

16 Dhoot NM, Kumar V, Shinagare A, Kataki AC, Barmon D and Bhuyan U: Evaluation of carcinoma cervix using magnetic resonance imaging: correlation with clinical FIGO staging and impact on management. J Med Imaging Radiat Oncol 56: 5865, 2012. PMID: 22339747. DOI: 10.1111/j.17549485.2011.02333.x

17 Kraljevic Z, Viskovic K, Ledinsky M, Zadravec D, Grbavac I, Bilandzija M, Soljacić-Vranes H, Kuna K, Klasnić K and Krolo I: Primary uterine cervical cancer: correlation of preoperative magnetic resonance imaging and clinical staging (FIGO) with histopathology findings. Coll Antropol 37: 561-568, 2013. PMID: 23941005.

18 Dappa E, Elger T, Hasenburg A, Düber C, Battista MJ and Hötker AM: The value of advanced MRI techniques in the assessment of cervical cancer: A review. Insights Imaging 8: 471-481, 2017. PMID: 28828723. DOI: 10.1007/s13244-017-0567-0

19 Schreuder SM, Lensing R, Stoker J and Bipat S: Monitoring treatment response in patients undergoing chemoradiotherapy for locally advanced uterine cervical cancer by additional diffusion-weighted imaging: A systematic review. J Magn Reson Imaging 42: 572-594, 2015. PMID: 25346470. DOI: 10.1002/jmri.24784

20 Genovesi D, Filippone A, AusiliCèfaro G, Trignani M, Vinciguerra A, Augurio A, Di Tommaso M, Borzillo V, Sabatino F, Innocenti P, Liberatore E, Colecchia G, Tartaro A and Cotroneo AR: Diffusion-weighted magnetic resonance for prediction of response after neoadjuvant chemoradiation therapy for locally advanced rectal cancer: Preliminary results of a monoinstitutional prospective study. Eur J Surg Oncol 39: 10711078, 2013. PMID: 23953231. DOI: 10.1016/j.ejso.2013.07.090

21 Delli Pizzi A, Cianci R, Genovesi D, Esposito G, Timpani M, Tavoletta A, Pulsone P, Basilico R, Gabrielli D, Rosa C, Caravatta L, Di Tommaso M, Caulo M and Filippone A: Performance of diffusion-weighted magnetic resonance imaging at $3.0 \mathrm{~T}$ for early assessment of tumor response in locally advanced rectal cancer treated with preoperative chemoradiation therapy. Abdom Radiol 43: 2221-2230, 2018. PMID: 29332248. DOI: $10.1007 / \mathrm{s} 00261-018-1457-8$ 
22 Delli Pizzi A, Caposiena D, Mastrodicasa D, Trebeschi S, Lambregts D, Rosa C, Cianci R, Seccia B, Sessa B, Di Flamminio FM, Chiacchiaretta P, Caravatta L, Cinalli S, Di Sebastiano P, Caulo M, Genovesi D, Beets-Tan R and Basilico $\mathrm{R}$ : Tumor detectability and conspicuity comparison of standard b1000 and ultrahigh b2000 diffusion-weighted imaging in rectal cancer. Abdom Radiol 44: 3595-3605, 2019. PMID: 31444557. DOI: 10.1007/s00261-019-02177-y

23 Liu Y, Bai R, Sun H, Liu H and Wang D: Diffusion-weighted magnetic resonance imaging of uterine cervical cancer. J Comput Assist Tomogr 33: 858-862, 2009. PMID: 19940650. DOI: 10.1097/RCT.0b013e31819e93af

24 Chen J, Zhang Y, Liang B and Yang Z: The utility of diffusionweighted MR imaging in cervical cancer. Eur J Radiol 74: 101106, 2009. PMID: 19442466. DOI: 10.1016/j.ejrad.2009.04.025

25 Haack S, Pedersen EM, Jespersen SN, Kallehauge JF, Lindegaard JC and Tanderup K: Apparent diffusion coefficients in GEC ESTRO target volumes for image guided adaptive brachytherapy of locally advanced cervical cancer. Acta Oncol 49: 978-983, 2010. PMID: 20831485. DOI: 10.3109/0284186X.2010.500619

26 Han K, Croke J, Foltz W, Metser U, Xie J, Shek T, Driscoll B, Ménard C, Vines D, Coolens C, Simeonov A, Beiki-Ardakani A, Leung E, Levin W, Fyles A and Milosevic MF: A prospective study of DWI, DCE-MRI and FDG PET imaging for target delineation in brachytherapy for cervical cancer. Radiother Oncol 120: 519-525, 2016. PMID: 27528120. DOI: 10.1016/j.radonc.2016.08.002

27 Olsen JR, Esthappan J, DeWees T, Narra VR, Dehdashti F, Siegel BA, Schwarz JK and Grigsby PW: Tumor volume and subvolume concordance between FDG-PET/CT and diffusion-weighted MRI for squamous cell carcinoma of the cervix. J Magn Reson Imaging 37: 431-434, 2013. PMID: 23023940. DOI: 10.1002/jmri.23830
28 Rosa C, Caravatta L, Delli Pizzi A, Di Tommaso M, Cianci R, Gasparini L, Perrotti F, Solmita J, Sartori S, Zecca IAL, Di Nicola M, Basilico R and Genovesi D: Reproducibility of rectal tumor volume delineation using diffusion-weighted MRI: Agreement on volumes between observers. Cancer Radiother 23: 216-221, 2019. PMID: 31109840. DOI: 10.1016/j.canrad.2018.10.004

29 Sun C, Chatterjee A, Yousuf A, Antic T, Eggener S, Karczmar G S and Oto A: Comparison of T2-weighted imaging, DWI, and dynamic contrast-enhanced MRI for calculation of prostate cancer index lesion volume: correlation with whole-mount pathology. Am J Roentgenol 212: 351-356, 2019. PMID: 30540213. DOI: $10.2214 /$ AJR.18.20147

30 Rischke HC, Nestle U, Fechter T, Doll C, Volegova-Neher N, Henne K, Scholber J, Knippen S, Kirste S, Grosu AL and Jilg CA: 3 Tesla multiparametric MRI for GTV-definition of dominant intraprostatic lesions in patients with prostate canceran interobserver variability study. Radiat Oncol 8: 183-194, 2013. PMID: 23875672. DOI: $10.1186 / 1748-717 X-8-183$

31 Ma DJ, Zhu J, Grigsby PW. Utility of FDG-PET in defining a high risk GTV for cervix cancer. Int J Radiat Oncol Biol Phys 75: S148-149, 2009. DOI: 10.1016/j.ijrobp.2009.07.351

Received March 17, 2020

Revised April 7, 2020

Accepted April 8, 2020 